\title{
Evaluation of an Internal Model Control Extension for Efficient Disturbance Rejection
}

\author{
Robin De Keyser, Clara M. Ionescu, Cosmin Copot \\ Ghent University \\ Department of Electrical energy, Systems and Automation \\ Technologiepark 913, 9052 Gent, Belgium \\ Robain.DeKeyser@UGent.be, ClaraMihaela.Ionescu@UGent.be, Cosmin.Copot@UGent.be
}

\begin{abstract}
This paper introduces an extension of the Internal Model Control algorithm for efficient disturbance rejection. The approach is based on ideas from model based predictive control and diophantine equation derivation. As an illustration of the power of the extension, an example from the process industry is borrowed, namely a drum boiler. The process is challenging for control since it has an integrator and non-minimum phase dynamics. The performance of the proposed extension is compared against nominal IMC design and PID. The simulation results suggest that the proposed algorithm outperforms the other implementations in terms of effective disturbance rejections.
\end{abstract}

\section{Introduction}

In the process industry, manipulating inputs to obtain a desired output in presence of temperature variations is an ubiquitous task for control [1]. Chemical reactors often require an additional process unit for temperature variation and reflux of outflow, namely a drum boiler. Naturalcirculation boilers are widely used in various chemical processing and related industries $[2,3]$. The design principle uses the difference in density between cooler water in the downcomer and the steam/water mixture in the riser to drive the steam/water mixture through the tubes. The boiler drum separates steam from water and contains inventory to accommodate operational changes. Water enters the riser tube, is heated, and undergoes a transition from a single-phase liquid to a mixture of saturated liquid and steam. As heat input increases, the proportion of steam vapor in the riser tube increases.

Maintaining the correct water level in the drum is critical for many reasons $[4,5]$. A water level that is too high causes flooding of the steam purification equipment; resulting in the carry over of water and impurities into the steam system. A water level that is too low results in a reduction in efficiency of the treatment and recirculation function. It can even result in tube failure due to overheating from lack of cooling water on the boiling surfaces. Typically, drum level is expected to be held within $2-5 \mathrm{~cm}$ of the set-point with some tolerance for temporary load changes.

This process has exotic dynamics due to the physical phenomena taking place in the drum. Under boiling conditions, steam supporting field products such as bubbles exist below the water/steam level interface. These bubbles have volume and therefore displace water to create a mis-representation of the true water level in the drum. Another effect upon drum level is pressure in the drum. Because steam bubbles compress under pressure (if the drum pressure changes due to load demands), the steam bubbles expand or contract respective to these pressure changes. A higher steam demand will cause the drum pressure to drop, and the steam bubbles to expand to give the appearance of a water level higher than it truly is. This fictitious higher water level causes the feedwater input to be shut down at a time when more water is really required. A surge in water level as a result of the drum pressure decreasing is called 'swell'. A water level decrease due to drum pressure increase is called 'shrink'. From control standpoints this is a challenging process.

Internal Model Control (IMC) techniques are part of model based control techniques which are often used in chemical process control. It has the potential to achieve good closed loop performance, while taking into account the model structure of the process (i.e varying time delays and periodic disturbances). Many successful implementations of IMC in real life processes have been reported $[1,6,7]$. In this paper we investigate the potential of the IMC as an integrated solution to reject effectively the disturbances at the input of the process. To evaluate the proposed extension for disturbance rejection we compare its performance against nominal IMC and PID control on the drum boiler process.

The paper is organized as follows: in Section 2 a description of a drum boiler system is presented. In Section 3 the IMC controller and the new extension of the IMC filter is presented. The results of the IMC controller, along 
with some implementation aspects followed by the experimental outcomes are presented in Section 4. A conclusion section summarizes the main outcome of this work.

\section{Process description}

The type of drum boiler we are attempting to reproduce in the form of a software simulator is a water-wall boiler which has radial tubes on three sides of the firebox, like in the Figure1. During operation, the tubes exposed to the

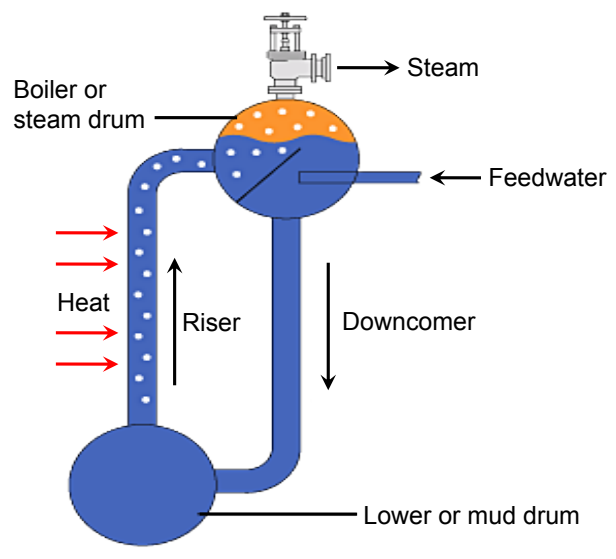

Figure 1. Drum boiler schematic represen-
tation

radiant heat from the flame are always producing steam. As the steam rises in the tubes, boiler water is also carried upward and discharged into the steam drum. Tubes that are not producing significant steam flow have a net downward flow of boiler water from the steam drum to the mud drum. Feedwater is pumped into the feedwater inlet by the use of a pump which has an input flow denoted $q_{f}(t)$. Also, steam demand from the system is increased or decreased by the use of a steam valve sucking in steam at the outlet of the drum for the turbine. The steam demand is always commanded as an outside disturbance by the turbine in the industrial power plant.

A common problem for this kind of dynamic system is dynamic shrink/swell, which are phenomena that produce variations in the level indication of the liquid surface in the steam drum whenever boiler load occur. The way in which the average level $L(t)$ inside the drum is measured is, again, an important reason for the shrink and swell dynamics which will be approximated by non-minimum phase linearized dynamic models.

Quoting from [8], an important fact is stated with regards to the dynamic response of the drum boiler level. Maintaining liquid level in the boiler steam drum while varying the power output of the entire installation is the highest priority. It is critical that the liquid level remains low enough to guarantee that there is adequate disengaging steam volume above the liquid, and high enough to assure that there is water present in every steam generating tube in the boiler. These requirements typically result in a narrow range in which the liquid level must be maintained. The feedwater used to maintain liquid level in industrial boilers often comes from multiple sources and is brought up to steam drum pressure by pumps operating in parallel. With multiple sources and multiple pumps, the supply pressure of the feedwater will change over time. Every time supply pressure changes, the flow rate through the valve, even if it remains fixed in position, is immediately affected. In order to have a better understanding of the physical inputs and outputs of the drum boiler, in Figure 2 there is shown a systemic-view with the four inputs and the two outputs of the drum boiler simulator.

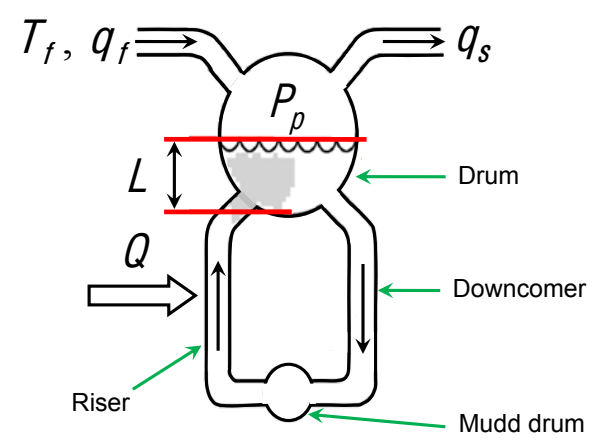

Figure 2. Drum boiler inputs/outputs - systemic view

Manipulated inputs:

$q_{f}(t)$ feedwater mass flow rate, in $\mathrm{Kg} / \mathrm{s}$

$Q\left(q_{p}(t)\right)$ heat flow to the burners in $\mathrm{kJ} / \mathrm{s}$, which is a function of the petroleum flow to the burners, $q_{p}$, in $\mathrm{Kg} / \mathrm{s}$

Disturbances:

$q_{s}(t)$ steam mass flow rate, in $\mathrm{Kg} / \mathrm{s}$ (unmeasured)

$T_{f}(t)$ temperature of the feedwater in $C^{\circ}$ (unmeasured)

Controlled outputs:

$L(t)$ level of the water in the drum, in $\mathrm{m}$

$P_{p}(t)$ pressure of the saturated steam in the drum, in bar

The complete set of modelling equations has been obtained in [8], which describes a physical drum boiler (the P16-G16 plant) operating in Sweden.

The linearized transfer function which describes the variation of the deviation level output of the boiler, $\delta L(t)$, with regards to the deviation feedwater flow input $\delta q_{f}(t)$ is shown in the next equation:

$$
P(s)=\frac{-0.0033004(s+0.3942)(s-0.09392)}{s(s+0.1488)(s+0.08333)}
$$

the feedwater flow $q_{f}(t)(\mathrm{kg} / \mathrm{s})$ has the biggest influence over the level output of the system, $L(t)$, which is also a logic and intuitive conclusion that can be drawn about the drum boiler simulator.

The level $L(t)$ has a very unusual variation as it resembles a non-minimum phase system with one nonminimum phase zero. Hence, the first tendency of the 
level inside the drum is to go in the opposite direction, then in the right direction. This fact is caused by the volume of steam inside the drum $V_{s d}(t)$. The latter first increases due to increased flow rate from the riser tubes, then decreases because of the increased pressure which rises and causes condensation of the steam.

Transfer function (1) contains one non-minimum phase zero which conveys quite intricate dynamics to the level output with respect to its most coupled input. As already presented, these types of non-minimum phase dynamics imply that the level control is not a problem suited for classical control strategies. A typical PID controller is not capable of accurately controlling the level variation inside the drum and to reject efficiently the disturbances at the input of the process. Another type of solution has been chosen which is model based: the IMC controller.

\section{Internal Model Control}

Internal model control (IMC) belongs to the class of model-based controllers [1]. Most of the algorithms are based on the use of a plant model, which captures the main process characteristics and allows a proper compensation/prediction of the process output, followed by an algebraic formulation that provides the optimal manipulated variables. In this section, a brief introduction to IMC is given $[6,7]$ along with the proposed extension for efficient disturbance rejection.

\subsection{Principles of the IMC formulation}

In a discrete-time formulation, the objective of a model-based controller is to use the process input sequence at each sampling instant, calculating an inverse function which intends to compensate for the process dynamics. Usually, the process model is a nonlinear dynamic relationship between the process output $y$ and the manipulated process input $u$ (i.e. $y(t)=f[y(t-$ $1), \ldots, u(t-1), \ldots])$. However, IMC assumes available a linear approximation of the process dynamics.

A closed loop control scheme is depicted in Figure 3(a), where $R$ denotes the controller and $P$ the process. Within the IMC context, the controller is designed based on compensating the process dynamics while ensuring a desired closed loop performance trajectory.

In order to make use of the inverse of the process to compensate dynamics, one must split the process in an invertible (good) part and a non-invertible (bad) part. This implies that if the process has RHP zeros, time delays or unstable poles in the transfer function, they are part of the non-invertible (bad) part of the process, i.e. $\hat{P}_{b}\left(q^{-1}\right)$. The remaining, invertible (good), part of the process is then denoted by $\hat{P}_{g}\left(q^{-1}\right)$ and is a causal and stable transfer function.

A 'basic' IMC filter, designed to follow step changes in the setpoint $w$ and to reject step disturbances $d$ at the

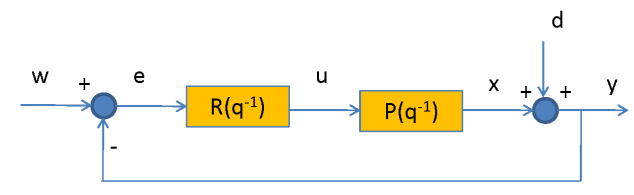

(a)

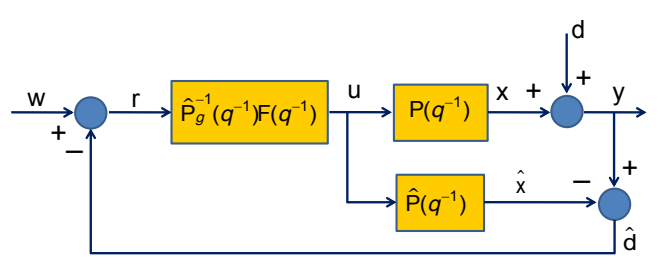

(b)

\section{Figure 3. (a) Schematic overview of the closed loop control scheme; (b) Schematic overview of the IMC structure}

output of the process, is given by:

$$
F\left(q^{-1}\right)=\frac{(1+a)^{n}}{\left(1+a \cdot q^{-1}\right)^{n}}
$$

with steady state gain $F(1)=1$ and $a$ a design parameter defined as $a=-e^{-T_{s} / \lambda}$. The (negative) values of this design parameter are in the range $0<<|a|<1$ and it is related to the closed-loop speed: if $\lambda$ is bigger, the $|a|$ is closer to 1 , the settling time will be bigger.

An 'extended' IMC filter, designed to follow a ramp setpoint and to reject ramp disturbances at the output of the process, is given by:

$$
F\left(q^{-1}\right)=\frac{(1+a)^{n}}{\left(1+a \cdot q^{-1}\right)^{n}} \cdot\left(1-f+f q^{-1}\right)
$$

with

$$
f=\frac{n a}{1+a}-\left(p_{1}+2 p_{2}+3 p_{3} \ldots+m p_{m}\right)
$$

and the parameters $p_{i}$ the coefficients of the bad part of the process:

$$
\left\{\begin{array}{l}
\hat{P}_{b}\left(q^{-1}\right)=0+p_{1} q^{-1}+p_{2} q^{-2}+\ldots+p_{m} q^{-m} \\
\sum_{i=1}^{m} p_{i}=1=P_{b}(1)
\end{array}\right.
$$

\subsection{Proposed extension}

The idea introduced here is based on modelling principles borrowed from model based predictive algorithms $[9,10]$. In the model based predictive control formulation [11], if the main frequency of the disturbance is known (measurable), it is possible to model it and provide this information to the controller. For instance, if a repetitive (e.g. sinusoidal) disturbance is present in the system, this 
can be modelled and used as a filter in the IMC algorithm. This can be best viewed in Figure 4, where the nominal closed loop can be defined as in the next equation based on the IMC control structure (Figure 3(b)) and on the fact that $P\left(q^{-1}\right)=P_{g}\left(q^{-1}\right) P_{b}\left(q^{-1}\right)$

$$
\begin{aligned}
& Y\left(q^{-1}\right)=P_{b}\left(q^{-1}\right) F\left(q^{-1}\right) W\left(q^{-1}\right)+ \\
& +\left[1-P_{b}\left(q^{-1}\right) F\left(q^{-1}\right)\right] \frac{C\left(q^{-1}\right)}{D\left(q^{-1}\right)}
\end{aligned}
$$

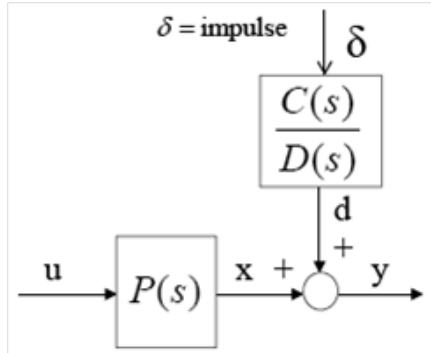

\section{Figure 4. Model of the disturbance effect}

According to the principle of the internal model control, the purpose is to remove the disturbance modes from the closed loop by including the generating polynomial $D\left(q^{-1}\right)$ in the controller. Hence, the numerator of $\left[1-P_{b}\left(q^{-1}\right) F\left(q^{-1}\right)\right]$ should include $D\left(q^{-1}\right)$ :

$$
\begin{aligned}
& F\left(q^{-1}\right)=\frac{F_{N}\left(q^{-1}\right)}{F_{D}\left(q^{-1}\right)} \Rightarrow \\
& \Rightarrow F_{D}\left(q^{-1}\right)-P_{b}\left(q^{-1}\right) F_{N}\left(q^{-1}\right)=D\left(q^{-1}\right) Q\left(q^{-1}\right)
\end{aligned}
$$

Notice that the poles of the disturbance filter are then indeed poles of the controller. Given $D\left(q^{-1}\right), F_{D}\left(q^{-1}\right)$ and $P_{b}\left(q^{-1}\right)$, one needs to find the polynomials $F_{N}\left(q^{-1}\right)$ and $Q\left(q^{-1}\right)$ using concepts of generalized disturbance rejection via the diophantine equation $[10,12,13]$ :

$$
P_{b}\left(q^{-1}\right) F_{N}\left(q^{-1}\right)+D\left(q^{-1}\right) Q\left(q^{-1}\right)=F_{D}\left(q^{-1}\right)
$$

Example: Consider a step disturbance $D\left(q^{-1}\right)=$ $\frac{1}{1-q^{-1}}$. Denote that $F_{D}\left(q^{-1}\right)=f_{0}^{D}+f_{1}^{D} q^{-1}+f_{2}^{D} q^{-2}+$ $\ldots$ and $P_{b}\left(q^{-1}\right)=p_{0}+p_{1} q^{-1}+p_{2} q^{-2}+\ldots$ then we have:

$$
\begin{aligned}
& \left(p_{0}+p_{1} q^{-1}+p_{2} q^{-2}+\ldots\right)\left(f_{0}^{N}\right)+ \\
& +\left(1-q^{-1}\right)\left(q_{0}+q_{1} q^{-1}+q_{2} q^{-2}+\ldots\right)= \\
& =f_{0}^{D}+f_{1}^{D} q^{-1}+f_{2}^{D} q^{-2}+\ldots
\end{aligned}
$$

Solving the diophantine equation leads to the following coefficient identification

$$
\left\{\begin{array}{l}
p_{0} f_{0}^{N}=f_{0}^{D} \Rightarrow f_{0}^{N}=\frac{f_{0}^{D}}{p_{0}} \\
q_{0}=f_{1}^{D}-p_{1} f_{0}^{N}
\end{array}\right.
$$

The order of $F_{N}$ and $Q$ has to be chosen such that the diophantine equation results in an unique solution. For this example, the filter used in order to prevent the steady-state error is given by: $F\left(q^{-1}\right)=\frac{f_{0}^{N}}{F_{D}\left(q^{-1}\right)}$. The polynomial coefficients of $Q$ are not further used. Applying the Laplace limit theorem to the closed-loop disturbance, results in:

$$
\lim _{s \rightarrow 0} s\left[1-P_{b}(s) F(s)\right] \frac{1}{s}=1-p_{0} \frac{F_{0}^{N}}{f_{0}^{D}}=0
$$

which shows indeed that the steady state error is zero.

We can now summarize that the controller $R\left(q^{-1}\right)$ for an IMC structure is given by:

$$
R\left(q^{-1}\right)=\frac{\hat{P}_{g}^{-1}\left(q^{-1}\right) F\left(q^{-1}\right)}{1-\hat{P}_{b}\left(q^{-1}\right) F\left(q^{-1}\right)}
$$

with $\hat{P}_{g}^{-1}\left(q^{-1}\right)$ the inverse of the (good) part of the process $\hat{P}\left(q^{-1}\right)$.

\subsection{Computer Aided PID Design: FRTool}

As a reference for the performance of the IMC controller, a classic PID control technique was also implemented and tested on the same process. The PID controller has been designed using a developed CAD-tool based on system frequency response. A PID controller is tuned with FRtool (A Frequency Response Tool for CACSD in Matlab) [14] designed in our laboratory and compatible with the Matlab platform. This computeraided-design software uses Nichols charts of the system in order to tune the controller, as given in Figure 5.

An important feature of a controller design tool is the possibility to define practically-meaningful design specifications -which will guide the designer in the tuning process. These specifications have to be converted to graphical restrictions to make the designer's job easier. Some of the traditional design specifications are gain margin (GM) and phase margin (PM). However, these specifications have not necessarily a clear physical meaning to a potential user (unless this user is e.g. a control engineer) - they are based on mathematical insight and system theory. Therefore, more practical specifications - which can be easily interpreted by any user - are settling-time and overshoot (\%OS) of the closed-loop time response, and of course robustness (Ro) of the design. Figure 5 shows the tuning of the PID controller on the process $P(s)$ with the specifications: $\% O S<25 \%, R o>0.5$ and settling time $T s<300$ seconds.

The textbook PID transfer function is: $K_{p}\left(1+\frac{1}{T_{i} s}+T_{d} s\right)=\frac{K\left(s+z_{1}\right)\left(s+z_{2}\right)}{s}$. The design of the PID is shown in Figure 5, with the controller gain $K=80$ and the controller's zeros $z_{1}=z_{2}=-0.025$. The corresponding PID parameters are given by: $K_{p}=4$, $T_{i}=80$ and $T_{d}=20$.

Notice that IMC has intrinsic disturbance filter rejection, while PID does not. Consequently, IMC can perform a perfect setpoint and perfect disturbance rejection simultaneously, while PID makes always a trade off between them.

\section{Results}

Since all systems to be controlled are digital systems, a discrete-time design and implementation of the control algorithm is meaningful. The discrete-time transfer func- 


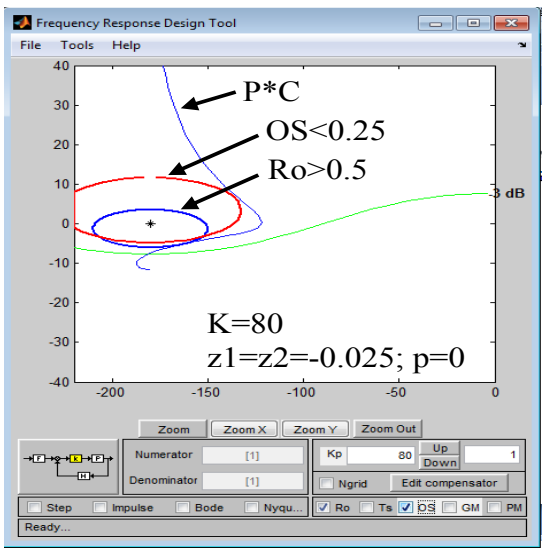

Figure 5. Tuning parameters for the PID controller using FRTool CAD based tuning package. A snapshot of the Nichols FRtool interactive window

tion of the drum boiler process $\hat{P}\left(q^{-1}\right)$ from (1) is:

$$
\frac{B\left(q^{-1}\right)}{A\left(q^{-1}\right)}=\frac{-0.01009 q^{-1}+0.0163 q^{-2}-0.00385 q^{-3}}{1-2.148 q^{-1}+1.917 q^{-2}-0.4982 q^{-3}}
$$

The good part of the process transfer function is

$$
\hat{P}_{g}\left(q^{-1}\right)=0.0033-0.001 q^{-1},
$$

whereas the bad part is

$$
\hat{P}_{b}\left(q^{-1}\right)=-3.0502 q^{-1}+4.0502 q^{-2} .
$$

Applying different disturbances at the input of the process, the proposed extension IMC (D-IMC) algorithm has been tested and validated using a drum boiler system.

The first disturbance applied is a step $\frac{C\left(q^{-1}\right)}{D\left(q^{-1}\right)}=$ $\frac{1}{\left(1-q^{-1}\right)}$ at the input of the process and it can be observed that the extended IMC and the new D-IMC have the same behavior. This can be also observed from Figure 6 where the extended and D-IMC are overlapping.

Figure 6 depicts the results for setpoint tracking and disturbance rejection closed loop performance for the IMC controllers and PID controller designed with FRTool. All controllers have similar results for setpoint tracking, while for the disturbance rejection, the nominal IMC cannot reject it and has steady-state error.

The second disturbance applied is a sinus with a bias equal to 1 , an amplitude $a=1$ and a frequency $\omega=$ $0.005 \mathrm{rad} / \mathrm{s}$. The disturbance is given by $\frac{C\left(q^{-1}\right)}{D\left(q^{-1}\right)}=$ $\frac{1}{\left(1-q^{-1}\right)^{2}\left(1-a e^{+j \alpha} q^{-1}\right)\left(1-a e^{-j \alpha} q^{-1}\right)}$ where $\alpha=\omega T_{s}$. The denominator $D\left(q^{-1}\right)$ of the disturbance is:

$$
1-3.994 q^{-1}+5.984 q^{-2}-3.984 q^{-3}+0.994 q^{-4}
$$

The denominator of the filter $F_{D}\left(q^{-1}\right)$ from (7) is given by:

$$
1-4 q^{-1}+6.4 q^{-2}-5.12 q^{-3}+2.04 q^{-4}-0.32 q^{-5}
$$

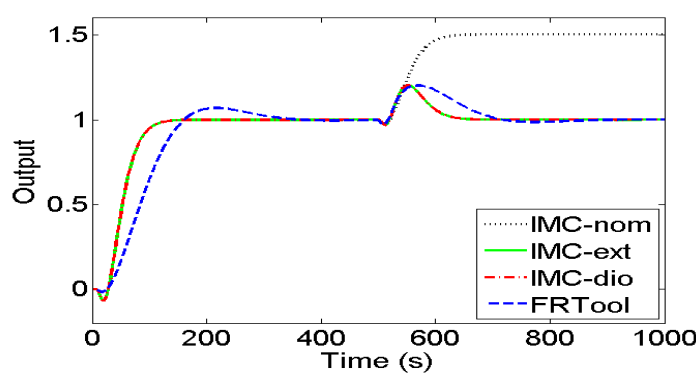

(a)

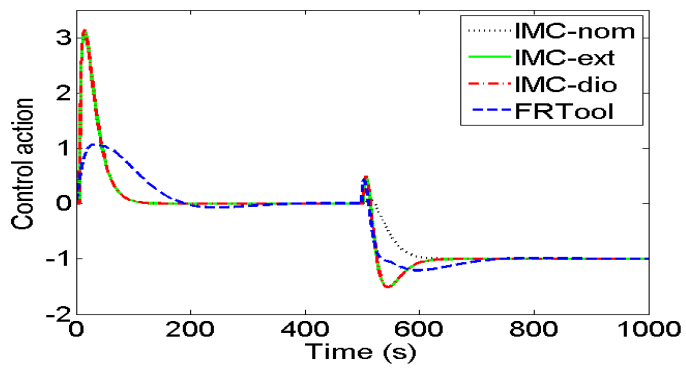

(b)

Figure 6. The setpoint tracking and disturbance rejection for all controllers: (a) the process output and (b) the control effort

Based on the diophantine equation from (8), we have a matrix form that:

$$
\begin{aligned}
& {\left[\begin{array}{cccccc}
0 & 0 & 0 & 0 & 1 & 0 \\
-3.05 & 0 & 0 & 0 & -3.99 & 1 \\
4.05 & -3.05 & 0 & 0 & 5.98 & -3.99 \\
0 & 4.05 & -3.05 & 0 & -3.98 & 5.98 \\
0 & 0 & 4.05 & -3.05 & 0.99 & -3.98 \\
0 & 0 & 0 & 4.05 & 0 & 0.99
\end{array}\right] \times} \\
& \times\left[\begin{array}{c}
f_{0}^{N} \\
f_{1}^{N} \\
f_{2}^{N} \\
f_{3}^{N} \\
q_{0} \\
q_{1}
\end{array}\right]=\left[\begin{array}{c}
1 \\
-4 \\
6.4 \\
-5.12 \\
2.04 \\
-0.32
\end{array}\right]
\end{aligned}
$$

from where the unknown coefficients can be determined:

$$
\left\{\begin{array}{l}
f_{0}^{N}=0.7106 \\
f_{1}^{N}=-2.0245 \\
f_{2}^{N}=1.9261 \\
f_{3}^{N}=-0.6119 \\
q_{0}=1 \\
q_{1}=2.1622
\end{array}\right.
$$

For the extended IMC the order of the filter is equal to 3 , while for the proposed D-IMC the order of the filter is $n=5$. Both these filters have zeros, hence they will produce high overshoot values for step setpoint changes. A solution is to use a pre-filter on the setpoint and cancel the zeros of the controller given by (12). Obviously this 
will lead to different dynamics.

The results for setpoint tracking and disturbance rejection when a sinus disturbance was applied are illustrated in Figure 7. Analyzing the output it can be observed that the nominal filter cannot reject the disturbance while in case of the extended IMC and the PID controller, the amplitude of oscillation are smaller. The disturbance is rejected without oscillations and zero steady state if D-IMC is used.

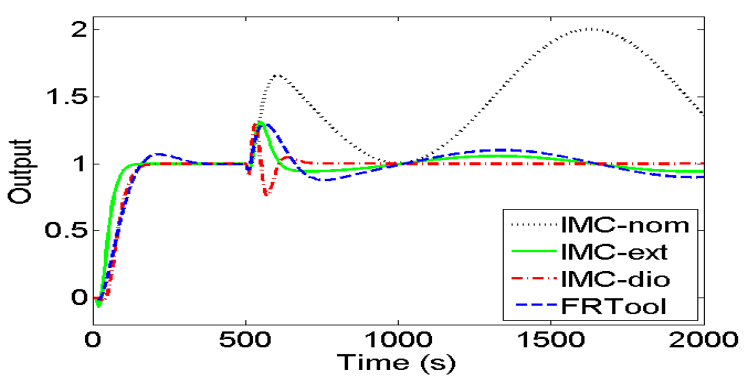

(a)

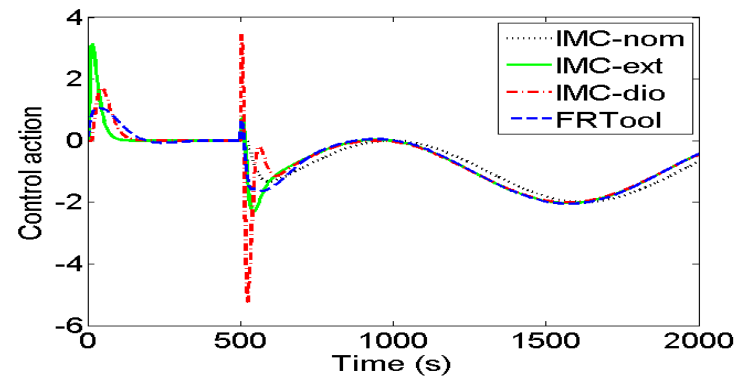

(b)

\section{Figure 7. The setpoint tracking and distur- bance rejection for all controllers when a si- nus disturbance is applied: (a) the process output and (b) the control effort}

The PID controller and the extended IMC has the same performances, while with slightly bigger effort it can be obtain remarkable improvements (disturbance rejection without oscillation). Notice that a better result can be obtained by the D-IMC if the dynamics of the plant are also compensated.

\section{Conclusion}

In this paper, an extension for effective disturbance rejection in internal model control has been presented and validated in simulation on a challenging process, i.e. the drum boiler. Results suggest that our method is suitable for filtering disturbances and next step is to test this into practice. Notice that a natural extension of the disturbance rejection problem is that of poorly damped systems, e.g. a flexible beam, a mass-spring-damper setup. For these highly oscillatory systems, the filter can contain the complex conjugated poles of the process transfer function in order to avoid oscillations in the output, leading thus to a damped response.

\section{References}

[1] B.W. Bequette, Process Control: Modelling, Design and Simulation. Pretice-Hall, NJ, 2003.

[2] J.B. Riggs and M.N. Karim, Chemical and BioProcess Control. Ferret Pub, 2007.

[3] G. Stephanopoulos, Chemical Process Control: An Introduction to Theory and Practice. Pretice-Hall, Upper Saddle River, NJ, 1984.

[4] M. Iacob and G. Andreescu, Drum-boiler control system employing shrink and swell effect remission in thermal power plants. 3rd Int. Congress on $\mathrm{Ul}$ tra Modern Telecommunications and Control Systems and Workshops, Budapest, pages 1-8, 2011.

[5] R. Cori and C. Maffezzoni, Practical-optimal control of a drum boiler power plant. Automatica, 20(2), pages 163-173, 1984.

[6] D.E. Rivera, M. Morari, S. Skogestad, Internal Model Control 4. PID Controller Design, Ind. Chem. Process Des. Dev., 25, pages 252-256, 1986.

[7] M. Morari, E. Zafiriou, Robust Process Control. Prentice-Hall, Eaglewood Cliffs, NJ, 1989.

[8] K.J. Åström, R.D. Bell, Drum-boiler Dynamics. Automatica, 36, pages 363-378, 2000.

[9] R. De Keyser, Model Based Predictive Control. Invited Chapter in UNESCO Encyclopaedia of Life Support Systems (EoLSS). Article contribution 6.43.16.1, Eolss Publishers Co Ltd, Oxford, ISBN 0 9542989 18-26-34 (www.eolss.net), 30p, 2003.

[10] E.F. Camacho and C. Bordons, Model Predictive Control. Springer- Verlag, London, 2004.

[11] R. De Keyser and C.M. Ionescu The Disturbance Model in Model Based Predictive Control. IEEE Conference on Control Applications, Istanbul, Turkey, CD paper CF-001472, 6p, 2003.

[12] M.J. Maciejowski, Predictive Control with Constraints. Prentice Hall, 2002.

[13] S.J. Qin and T.A. Badgwell, A Survey of industrial model predictive control technology. Control Engineering Practice, April 2002.

[14] R. De Keyser, C. Ionescu, FRtool: a frequency response tool for CACSD in MatLab. in Proceedings of IEEE Conf on Computer Aided Control Systems Design (CACSD-CCA-ISIC), Munchen, Germany, pages 2276-2280, 2006. 